\title{
Problems in Industrial Structure of International Tourism in Guangdong Province and Its Optimization
}

\author{
Hui Li \\ Tourism School \\ Zhuhai College of Jilin University \\ Zhuhai, China
}

\begin{abstract}
This paper is guided by the theory of industrial structure optimization, aiming at promoting the leap of Guangdong's international tourism industry. By analyzing the status quo and problems of the industrial structure in the development of Guangdong's international tourism, combining with the product structure of Guangdong's international tourism and the structure of its source market, this paper has made in-depth analysis of the characteristics of Guangdong's international tourism. It has put forward some specific optimization suggestions for the structure of Guangdong's international tourism.
\end{abstract}

Keywords-Guangdong Province; international tourism; industrial structure

\section{INTRODUCTION}

Inbound tourism is an important part of China's tourism industry and an important manifestation of the development of the tourism industry. Guangdong International Tourism has always been a leader in the country, both in terms of the number of inbound tourists and foreign exchange earnings. While Guangdong international tourism industry has made considerable progress, its internal structural contradictions have become increasingly prominent. The outstanding performance is the imbalance of industrial factors development, the imbalance of internal development of various factors and the imbalance of regional tourism development, which restricts the improvement of its overall quality and efficiency. The adjustment of economic structure will be a major issue that needs to be resolved in the development of tourism in Guangdong province.

\section{PROBLEMS IN THE INDUSTRIAL STRUCTURE OF GUANGDONG INTERNATIONAL TOURISM}

Through the analysis of the status quo of the industrial structure of Guangdong international tourism and comparative analysis on many aspects, it can be seen that Guangdong international tourism has a considerable scale and a relatively complete structural system. Further adjustment and optimization of the internal structure of each department is underway. The structure has shown a trend of evolution towards rationalization and heightening. However, this evolutionary trend is not very significant. The process of structural transformation is relatively slow, forming a relatively sharp structural contradiction. In the context of the product structure and the structure of the source market according to the analysis path of "industry internal structure - structural benefit - structural productivity - structural relevance", the outstanding contradictions and problems in the international tourism in Guangdong province are shown as the followings:

\section{A. Low-level Specialization}

The overall professionalization of Guangdong international tourism is relatively low, and the differences between departments are large. Among the specialized sectors, the advantages of the transportation sector are outstanding. Among the non-specialized sectors, the specialization varies greatly from year to year, showing the characteristics of volatility development and insufficient stability. The transportation sector has obvious advantages. The specialization of accommodation and catering is slightly higher than that of the national average, forming the interannual instability of the development of the two sectors. The entertainment and tourism have good development in recent years. However, the specialization is not high. There is the largest gap between the six sectors and the commodity sales department in the term of national average. It is the department with the worst professional level. In short, the overall professionalization level of Guangdong international tourism is not high, which makes the driving force for the development of Guangdong international tourism insufficient Therefore, from the perspective of giving full play to the advantages of regional resources, the specialization and scale of Guangdong international tourism needs to be further improved.

\section{B. Poor Structural Stability}

Since the 1990s, the changes in the structure of Guangdong international tourism have been in volatility development. Sometimes, the fluctuations between years are also large. For the development trajectory of each department, whether it is the proportion of its foreign exchange earnings from tourism or its respective elasticity coefficient and specialization level, the fluctuation is more prominent than that of the whole country. From the index of measuring the stability of the tourism industrial structure, and the change index of the industrial structure, the volatility of structural changes is large. Due to the uncoordinated relationship among the departments of Guangdong 
international tourism and the imbalance of development, the stability of industrial structural system of Guangdong international tourism is poor. The entropy of industrial structural system of Guangdong international tourism has a large decline. There is attenuation in the stability of industrial structure, affecting the overall utility.

\section{Change of Industry Structure Has Little Effect on the Tourism Industry}

The change of industrial structure is closely related to the growth of economic aggregate. As a comprehensive economic industry, the industrial structure directly affects the total growth of tourism industry. And it has decisive influence on the development and economic benefits of tourism industry. In the process of analyzing the structural productivity of Guangdong international tourism, through the calculation of the change index of industrial structure and the coefficient of productivity structure, the structural changes of Guangdong international tourism have been more volatile since the 1990s. On the one hand, the development of Guangdong international tourism promotes the active participation and development of various sectors of tourism. On the other hand, it also reflects the impact of certain unreasonable factors in structural changes, such as a large proportion of traffic, and the decline in shopping and accommodation. And when analyzing the correlation between industry composition and foreign exchange earnings, we can see that from 1991 to 1996, the industrial structure change index of Guangdong international tourism industry structure in 2002 and 2004 is positively related to the change of growth rate of foreign exchange earnings of international tourism. The larger the change index is, the higher the growth rate will be. From 1997 to 2001, the change index of industrial structure is roughly negatively correlated with the growth rate of earning. The larger the industry structure change index is, and the relatively low growth rate will be. It can be seen that the structural changes of Guangdong international tourism are highly volatile; the changes in its industrial structure are not obvious in promoting the entire tourism industry. This further indicates that the unreasonable factors of structural changes are objective.

\section{Evolution of the Tourism Structure Towards Rationalization Is Not Obvious and the Structural Transformation Is Slow}

Judging from the changes in the composition of tourism foreign exchange earnings in Guangdong Province, the gap in the ability to earn foreign exchange in various sectors of the tourism industry has been in a state of volatility. Longdistance transportation and accommodation have always been in the top two. The three sectors of "food, housing and transportation" have experienced fluctuations in recent years. Tourism and entertainment have increased slightly, while the shopping sector has been lower than the national average. In general, the traditional "food, housing, and transportation" have a tendency to decline. The trend of diversification and decentralization of share distribution is not very obvious. The dominant position of foreign exchange earning in rigid sectors such as long-distance transportation and accommodation has not been replaced by the benign development momentum of commodity sales and entertainment. The optimization of tourism industry structure is not obvious, and the trend of tourism industry structure to rationalization is not obvious. The transformation process of the entire tourism industry structure is relatively slow.

\section{E. The Contribution Rate of Industrial Structural Benefits of Tourism Is Low}

The industrial structure of Guangdong international tourism does not have the advantages. The structure does not contribute much to the economic growth, and the industrial structure needs to be adjusted. In terms of growth rate, structural contribution and competitiveness, in recent years, the best-developed sector is the tour department. This will become a reasonable direction for the future development of Guangdong international tourism industry, followed by the entertainment sector. Tour, purchase, and entertainment have become the growth sectors with departmental advantages. However, the shopping sector is the sector with the largest gap from the national average. Although the gap has decreased in recent years, it still needs to be further improved. The transportation, accommodation and catering departments have a good foundation. However, the development momentum is insufficient in the later period. Transportation and accommodation are the growth sectors. However, they do not have the advantage of the department. They are at a competitive disadvantage. The status of catering with poor competitiveness is declining year by year. In the basic position of the tourism industry, the adjustment of the department is very urgent, especially the catering sector.

\section{F. The Degree of Correlation Among Internal Structures of Tourism Industry Structure Is Relatively Low}

The degree of correlation among the departments of Guangdong international tourism is generally not high. Due to human obstacles and industrial barriers, the objective economic linkages among the various systems within the tourism industry are not very close, making it difficult to achieve integrated management. There is a lack of coordination among the elements. At the same time, there is a large difference in the degree of relevance between the departments, which makes the tourism industry more dependent on the accommodation and catering departments. Moreover, in recent years, the development status and competitiveness of these two departments have declined, which has led to the overall fragility of the industrial structure of tourism.

\section{THE GENERAL PRINCIPLE OF STRUCTURAL OPTIMIZATION OF INTERNATIONAL TOURISM INDUSTRY}

\section{A. The Principle That the Tourism Supply Structure Is Compatible with the Structure of Tourism Demand}

Under the conditions of market economy, the supply of tourism products is based on the needs of tourists. The direction, variety, quality and structure of tourism supply should be established and adjusted around the direction, 
variety, quality and structure of tourism demand. The destination country or region must conduct in-depth investigation and research on the tourism market in each period, grasp the changing trend of tourism demand in each period, and adjust the content and structure of its supply accordingly to make the two basically adapt to each other.

\section{B. The Principle of Coordinating Leading Industries with Related Industries}

In many industries related to tourism, travel agencies are responsible for the tourism production organization and sales. Its role runs through the tourism activities. Its development level determines the rise and fall of a series of other related industries. It should first determine the dominant position of travel agencies. In contrast to the leading industries, other industries directly or indirectly related to tourism activities are related industries. And the products and services they provide must be combined by travel agencies to form tourism products. Therefore, in the process of rationalizing the structure of the tourism industry, it is necessary to highlight the dominant position of the travel agency industry, and also to strengthen the coordination between the related industries and the leading industries and the coordination among the related industries.

\section{The Principle That the Regional Tourism Industry Structure Is in Line with the International Tourism Industry Structure}

In the international tourism industry, with the intensification of competition in the world tourism market, some developed countries that have a large number of tourists entering and leaving the business have successively formulated and implemented a series of stricter standards and norms for tourism supply. The World Tourism Organization also makes efforts to promote uniform technical standards for tourism products. This indicates that the world tourism economic structure is changing to a higher level. It requires the tourism industry structure of destination countries, especially developing countries, to be linked to the world trade product trade structure, and the standards of increasingly strict international tourism products. Only in this way can we maintain and expand our share of the international tourism market.

\section{OPTIMIZATION OF INDUSTRIAL STRUCTURE OF GUANGDONG INTERNATIONAL TOURISM}

According to the analysis and problems of the industrial structure of current international tourism in Guangdong province, combined with the objectives and principles of tourism industry structure optimization, some suggestions for optimizing the tourism industry structure are proposed:

\section{A. To Strengthen Sales and Attract More Tourists from Developed Countries}

The level of national economic development of the source country (region) is directly related to the leisure time and income of tourists, which directly affects the consumption level of tourists. Generally speaking, the higher the level of national economic development is, the greater the income and leisure time will be. This will stimulate the improvement of consumption. Therefore, the level of national economic development in the source countries is the most fundamental factor affecting the consumption structure. Therefore, while strengthening publicity and promotion to ensure the stable development of overseas source markets, we should strive to attract more tourists from developed countries.

\section{B. To Promote the Improvement of the Tourism Industry Structure by Virtue of the Advantages of Exhibitions}

The development of convention and exhibition economy in Guangdong Province, especially in the Pearl River Delta region, is very rapid. On the whole, the cities in the Pearl River Delta Convention and Exhibition Economic Industry Zone will develop various types of exhibitions according to their respective characteristics, which will form a multi-level and complementary exhibition market structure. Guangzhou, as the central city of the South China Convention and Exhibition Industry, will continue to hold the large-scale comprehensive exhibition such as "Canton Fair", which is mainly based on large scale and exhibitors. Shenzhen holds high-tech professional exhibitions. The other cities in the Pearl River Delta rely on characteristic industries to hold exhibitions with strong industrial colors, such as Human clothing festival, the People's Fair in Dongguan, etc. Moreover, there is obvious interaction between the exhibition economy and the tourism economy. Organizing conferences and exhibitions, especially international ones, must involve six elements of food, housing, travel, postal, purchase and entertainment. The participants of various domestic and international exhibitions also naturally form an important source for the tourism industry.

Therefore, we should rely on the advantages of the development of Guangdong exhibition economy to further promote the development of international tourism. Through the further development of exhibition tourism, we will vigorously promote the entry of overseas tourists, increasing the international tourist source of Guangdong Province. The increase of international tourists, especially the increase of business sources with higher consumption levels, can greatly improve the economic benefits of international tourism. And the improvement of economic benefits is also of great significance to the optimization of tourism industry structure.

\section{To Improve the Internal Structure of the Factors and the Structural Benefits of the Tourism Industry}

Basic elements: (1) Transportation: It should provide more convenient and efficient services through travel ecommerce and network reservation system. We can gradually improve the transportation system among central cities and major scenic spots, and strengthen the construction of supporting facilities for transportation systems, improve the levels of special vehicles for tourism and the service quality of tour guides and passengers. (2) Tourism landscape: It can give play to the advantages of resources, strengthen product features, strengthen the development and construction of branded products and new tourism products, 
and strengthen the construction of tourism networks. It can control the development scale of the tour department, attach importance to the improvement of the quality of tourism products, support the development of special tourism products, and increase marketing efforts. (3) Catering: It can excavate cultural characteristics, highlighting scale effects. It can strengthen the cultural packaging of catering products. In the development of catering products, we focus on excavating national culture and local traditional culture. We implement omni-directional packaging of catering products and increase the added value and attractiveness of catering products. We also excavate local famous foods, improve the craftsmanship of traditional brands, and constantly introduce new brands. (4) Accommodation: It forms a reasonable spatial layout and product system, and adds special hotels. On the basis of the existing hardware facilities and software advantages, we pursue the combination of local culture and history, natural and luxurious design, and provide a variety of supply to meet different levels of demand.

\section{To Enhance the Diffusion Effect of Leading Industries and Achieve a Balance Between Supply and Demand}

In tourism economic activities, travel agencies are responsible for the organization and sales of tourism products. There is high-level correlation between other sectors of the tourism industry and the travel agency. It has the ability to drive and transform other industrial sectors. Its role runs through tourism activities. The development has always determined the rise and fall of a series of other related industries, affecting the development level of the entire tourism industry. Therefore, in the process of optimizing the structure of tourism industry, the dominant position of travel agencies should be established first. Establishing the dominant position of travel agencies in the tourism industry is an important hub for the upgrading of the tourism industry structure.

\section{E. To Improve the Scientific and Technological Content and Promote the Upgrading of Tourism Industry Structure}

Scientific and technological progress is the most active factor affecting the change of industrial structure. It is the key to promoting the improvement of social productivity and the development of industrial structure to a high level. The rapid development of modern tourism benefits by the rapid development of high-tech such as modern transportation, new materials and computer communication technology. Therefore, the tourism industry in Guangdong can speed up the high-tech transformation of various sectors of the tourism industry by establishing new information management systems such as tourist destination systems, computer reservation systems, and hotel management systems, improve the technological content and information content of products, adapt to the needs of consumers in the information age and industrial changes, and promote the development of the tourism industry structure.

\section{CONCLUSION}

The industrial structure of Guangdong international tourism is not reasonable enough and its height is not prominent enough, which reduces the aggregation level and transformation ability of tourism industry institutions, and restricts the pace of rapid and healthy development of Guangdong international tourism industry. The structural problems of the tourism industry have limited the growth rate of Guangdong international tourism industry, affecting the expansion of consumers' demand and the development potential of the tourism industry. The deviation of the industrial structure has affected the quality and efficiency of the economic growth of the entire tourism industry, which has also affected the improvement of its competitiveness. Therefore, through the optimization of product structure and customer source market structure, the optimization of the industrial sector structure and the improvement of structural benefits must be promoted. It will promote the further rationalization and high-level development of the tourism industry structure. The optimization of the entire tourism industry structure can be realized. And then, it can effectively promote the development of the tourism industry.

\section{REFERENCES}

[1] Du Yanan, Liu Jia, Li Yingying. Research on the relationship between tourism industry structure optimization and economic growth in coastal areas against the background of urbanization $[\mathrm{J}]$. Contemporary Economy, 2017 (31): 69-71.

[2] Yan Ying, Zhang Guanghai, Wang Qiong. The evolution of tourism industry structure of world heritage sites and its contribution to tourism economy_-Taking Qufu city of Shandong province as an example[J]. Regional Research and Development, 2017, 36(04): 105109.

[3] Fang Yelin, Huang Zhenfang, Hu Zui, Li Jinglong, Liu Fajian. The temporal and spatial pattern evolution and type division of inbound tourism industry structure in Mainland China $[\mathrm{J}]$. Economic Geography, 2016, 36(03): 179-185.

[4] Feng Weihong, Hu Jianling. Research on the relationship between network structure of tourism industry clusters and corporate performance [J]. Economic Issues, 2016 (02): 125-129.

[5] Yuan Yaoqing, Ren Peiyu. The upgrading mechanism and path of tourism industry structure in industry convergence domain[J]. Shandong Social Sciences, 2016(01):119-123.

[6] Li Hui, Kan Xinglong. Analysis of the structure benefit of guangdong international tourism [J]. Special Economic Zone, 2011 (09): 144-145. 